\title{
HIV Co-Testing Among Veterans with Chronic Hepatitis $C$ in the Veterans Health Administration
}

\author{
Bret E. Fuller ${ }^{*}, 1,2,3$, Veronica L. Rodriguez ${ }^{1}$, Alex Linke ${ }^{1}$ and Peter Hauser ${ }^{*}, 4$ \\ ${ }^{I}$ Portland VA Medical Center, Portland, Oregon 97239, USA \\ ${ }^{2}$ Oregon Health \& Science University Department of Public Health and Preventive Medicine, Portland, Oregon 97239, \\ USA \\ ${ }^{3} N W$ Hepatitis C Resource Center, VAMC, Portland, Oregon 97239, USA \\ ${ }^{4}$ VISN 22 Network Office; 300 Oceangate, Suite 700, Long Beach, California 90802, USA
}

\begin{abstract}
Objective: This retrospective study analyzed the testing rates of individuals enrolled in the Hepatitis C Clinical Case Registry for the Veterans Health Administration (VHA) in order to determine Human Immunodeficiency Virus (HIV) co-testing rates for veterans with hepatitis C (HCV). Design: A chart review of 247,006 veterans enrolled in the National HCV Clinical Case Registry was examined retrospectively. Regression analysis identified factors that increased the probability of being tested for HIV. Methods: Simple odds ratios and a complex regression were applied to this dataset to calculate testing incidence and prevalence of HIV. Results: Only approximately one third of veterans with HCV were tested for HIV and, of these, $13.2 \%$ were positive. Sixty eight percent of veterans with HCV were not co-tested for HIV and within this group there may be a significant number of unidentified cases of HIV. Veterans with severe mental illness, substance use disorders and marijuana abuse/dependence treatment were more likely to be tested for HIV. Antiviral therapy for HCV had no impact on likelihood of co-testing prior to treatment initiation. Conclusions: Most veterans with $\mathrm{HCV}$ are not co-tested for HIV despite common risk factors for HIV and HCV infection. Mandatory testing for HIV, at least among veterans with $\mathrm{HCV}$, as well as identification and removal of barriers to HIV testing within the VHA would serve to facilitate disease management for veterans in the future.
\end{abstract}

Keywords: HIV, hepatitis C, testing, co-testing, veterans.

\section{INTRODUCTION}

The hepatitis $\mathrm{C}$ virus (HCV) is a common blood-borne infection that infects approximately 3.9 million Americans and is one of the leading causes of liver disease in the United States. HCV is transmitted through contact or exposure with infected blood through mechanisms such as injection drug use, blood transfusions, intranasal cocaine use, and high risk sexual practices [1]. Although the general population prevalence of $\mathrm{HCV}$ is estimated to be $1.8 \%$, it has been reported to be up to four-fold higher among veterans using Veterans Affairs (VA) facilities. Studies have reported that the HCV infection rate among US veterans may be as high as $10.6 \%$ with the majority, $8.2 \%$ as chronic infections [2]. A national prospective study of patient sera calculated the prevalence of HCV among veterans who use Veteran's Health Administration (VHA) facilities and clinics for their health care to be $4.0 \%$ but with a non-participation weighting correction, the rate was estimated to be $5.4 \%$. In this particular study the characteristics of those infected included: income lower than $\$ 10,000$ per year $(17.1 \%)$, injection drug

Address correspondence to these authors at 3170 SW US Veterans Park Road, Mailcode: P3MHDC, Portland, OR 97210, USA; Tel: 03 220-8262, Ext. 54469; Fax: 503 220-3499;

E-mail: bret.fuller@va.gov; VISN 22 Mental Health Services Lead, VISN 22 Network Office, 300 Oceangate- Suite 700 Long Beach, CA 90822, USA; Tel: 562-826-8000, Ext. 2629;

E-mail- peter.hauser2@va.gov use or blood transfusion (30.2\%), and service in the Vietnam War (11.0\%). Other risk factors identified in this study were tattoos and body piercing, problem drinking, a high number of sex partners particularly those known to be injection drug users, and incarceration history. Further, $11.4 \%$ of those testing positive for $\mathrm{HCV}$ had a psychiatric illness documented in the electronic medical record a, 22.6\%, had a drug use disorder documented in the electronic medical record and $15.4 \%$ used marijuana [3].

Another infectious disease that affects veterans is HIV. The VHA treats more people for HIV infection than any other healthcare system in the US [4]. The most recent estimate of the overall prevalence of HIV in veterans was $1.49 \%$ [5]. The percentage of all HIV infected people who also have $\mathrm{HCV}$ ranges from $15 \%$ to $30 \%$ and one study reported that $37 \%$ of people with HIV were co-infected with HCV [6]. From a different perspective the percentage of all $\mathrm{HCV}$ infected people who have HIV ranges from 5\% to $10 \%$ [6] and among veterans with HCV the prevalence of HIV was estimated to be approximately $8.4 \%$ in those with identified risk factors that were tested [7]. A second study of US veterans in the Veterans Integrated Service Network in New Jersey and New York City reported a much higher coinfection rate of $24.8 \%$ [2].

One obvious explanation for the high incidence of coinfection is that HCV and HIV share common risk factors such as sharing needles and works for injection drug use, 
modes of transmission (blood to blood contact) and multiple sex partners. A higher rate of HIV and HCV co-infection is also associated with psychiatric diagnosis, substance use disorders and/or a severe mental illness [8-10].

Unrecognized HIV/HCV co-infection has serious implications on liver health as it may accelerate the progression of HCV liver disease [11]. People with HCV and HIV co-infection were twice as likely to develop cirrhosis [12]. Early detection of people co-infected with HIV and $\mathrm{HCV}$ is essential in managing treatment of these infections [13]. Also late detection of HIV has serious health consequences for both patients with as well as those without $\mathrm{HCV}$ including poor response to antiretroviral medications, earlier onset of AIDS-defining illnesses, and high mortality risk $[14,15]$. Late detection of HIV also leads to higher incidence rates as the transmission rate of people ignorant of their positive status is 3.5 times higher than those who are aware [16].

HCV/HIV Co-testing: A study of 293,455 VA medical records in Veteran Integrated Service Network 20 (VISN 20) consisting of 8 facilities and clinics in Alaska, Washington, Oregon and Idaho found that $32.8 \%$ of veterans served were tested for HCV, $5.5 \%$ were tested for HIV, and $4.3 \%$ were co-tested for both HCV and HIV [17]. Of those veterans who were tested for HCV and found to be HCV positive, only $35 \%$ were co-tested for HIV ( $4.9 \%$ were HIV positive). However among those tested for HIV and found to be positive for HIV, approximately $79.1 \%$ were co-tested for HCV. This disparity suggests that barriers to HIV testing may exist in Veterans Health Administration (VHA) facilities and clinics [17]. The purpose of this study was to examine the rates of co-testing among veterans who were in the VHA national HCV clinical case registry (HCV CCR) between 2001 and 2006 in an attempt to replicate and extend our previous findings from the VISN 20 regional database.

\section{MATERIALS AND METHODOLOGY}

This study examined the incidence of co-testing among veterans enrolled in the national HCV CCR. A retrospective analysis of veterans enrolled between 2001 and October 2006 was conducted. The HCV CCR is a national Veterans Health Administration (VHA) database in which all veterans who are included have chronic $\mathrm{HCV}$ and are viremic. It does not include veterans who receive healthcare outside of VHA facilities and clinics. Chronic HCV is defined as a positive $\mathrm{HCV}$ antibody test and Polymerase Chain Reaction (PCR) confirmation of viremia. In about $30 \%$ of patients with positive $\mathrm{HCV}$ antibodies, the virus has cleared and the person is no longer infected. Only veterans with PCR confirmation after positive $\mathrm{HCV}$ antibody testing are entered into the HCV CCR.

In $2001 \mathrm{HCV}$ screening and testing was mandated for all facilities and clinics in the VHA. Therefore the study period of 2001 to 2006 is of interest as it considers the effects of this policy change. All clinical contact ICD-9 codes were examined and extracted from these data. Veterans who were HIV and HCV positive prior to 2001 were included in this study. Veterans in the registry who died prior to 2001 or who did not have PCR confirmation of a positive HCV antibody test were excluded from the analysis. The variables examined were: HIV status, HIV testing, HIV test results, the number of clinical contacts for $\mathrm{HCV}$, and dichotomous indicators of any visit for the following conditions: bipolar disorder, schizophrenia, major depressive disorder, post traumatic stress disorder (PTSD), alcoholism and other alcohol use disorders, any substance use disorder (SUD), cirrhosis, chronic liver disease, non-alcoholic fatty liver disease (NAFLD), marijuana abuse/dependence treatment, diabetes, and hypertension. For each of these variables, the number of visits was dichotomized so that 0 visits was coded as a " 0 " and visits greater or equal to 1 was coded as a ' 1 '. The only variable that was not dichotomized was the number of HCV visits. Most of the participants had at least one visit for $\mathrm{HCV}$ which prompted their entry into the HCV clinical case registry. In order to capture the variability between patients with many hospital or clinic visits for HCV (those likely going through antiviral treatment) contrasted with patients with one or two visits for HCV (patients who have been tested and evaluated but not receiving antiviral therapy), the number of visits was entered into the regression equation.

Data Analysis: Estimates of the rate of testing and seroprevalence of HIV among the registrants in the HCV clinical case registry were conducted. Second, a logistic regression assessed the factors that contributed to the increasing probability that $\mathrm{HCV}$ patients were tested for HIV. The model was constructed with the dependent variable of whether the patient was tested for HIV (1) or not tested $(0)$ in the period of observation $(2001-2006)$. The first variable entered was the number of appointments attended for the HCV diagnosis. The second step simultaneously entered a set of categorical variables that indicated whether during the observation period, the patient had been seen for a particular medical condition. Finally, an analysis indicated whether being on antiviral or interferon treatment influenced HIV testing rates.

\section{RESULTS}

Demographics: The national sample was $97 \%$ male, consistent with the overall veteran population. African Americans made up about $22.7 \%$ of the sample. The average age of the sample was 56 years and about $9.4 \%$ of the sample died during the observation period. Table $\mathbf{1}$ displays the demographic variables and presence of psychiatric disorders and SUDs of all veterans in the HCV CCR and also compares veterans who are HIV- with those that are HIV +. Veterans were considered HIV- if they had not been tested or were tested and HIV-. It should be noted that those who are HIV + in this sample were those who were tested and identified as HIV + during the 2001-2006 time frame, or who were tested prior to 2001 and HIV +.

All but one of the variables showed statistically significant differences between groups because of the enormous sample size. HIV+ veterans had a lower mean age than veterans who were HIV-. A larger percentage of HIV + veterans were male and African American. Also a larger percentage of HIV+ veterans died during the observation period. SUDs and all psychiatric disorders were more common among veterans with HIV+ with the exception of PTSD.

Prevalence of HIV: For this study 247,006 patients were extracted from the HCV Clinical Case Registry between 
Table 1. Demographics of the Sample Overall and by HIV Infection

\begin{tabular}{|c|c|c|c|}
\hline Variable & $\begin{array}{c}\text { Overall Sample } \\
\mathbf{N}=\mathbf{2 4 7 , 0 0 6}\end{array}$ & $\begin{array}{c}\text { HIV-* } \\
\mathrm{N}=\mathbf{2 3 6 , 1 7 6}(95.6 \%)\end{array}$ & $\begin{array}{c}\text { HIV+ } \\
\mathrm{N}=\mathbf{1 0 , 8 3 0}(\mathbf{4 . 4 0 \% )})\end{array}$ \\
\hline Mean Age (years) & 55.74 & 55.84 & $53.45^{\wedge}$ \\
\hline Male (\%) & 96.98 & 96.92 & $98.22^{\wedge}$ \\
\hline Died (\%) & 9.40 & 9.10 & $15.90^{\wedge}$ \\
\hline African American (\%) & 22.70 & 21.60 & $45.4^{\wedge}$ \\
\hline Substance Use Disorder** (\%) & 62.31 & 61.66 & $76.50^{\wedge}$ \\
\hline Depression (\%) & 50.50 & 50.10 & $59.32^{\wedge}$ \\
\hline PTSD (\%) & 25.62 & 25.60 & 25.92 \\
\hline Schizophrenia (\%) & 12.38 & 12.10 & $18.32^{\wedge}$ \\
\hline Bipolar Disorder (\%) & 12.61 & 12.50 & $15.18^{\wedge}$ \\
\hline
\end{tabular}

*HIV- includes veterans who were tested for HIV as well as veterans who were never tested.

$* *$ SUD $=$ Substance Use Disorder.

$\wedge$ Significant at $\mathrm{p}<.001$.

2001 and October, 2006. This included 7,801 (3.2\%) of patients that had previous diagnoses for HIV before 2001. Slightly more than one third $(86,364 / 247,006$ or $35 \%)$ were tested for HIV or had a previous diagnosis for HIV before 2001. Of those who had no documentation of HIV infection as of $2001,78,563(32.8 \%)$ were tested for HIV. Of those tested for HIV, 3,029 (3.9\%) were HIV positive. The overall HIV rate among the HCV CCR participants who were tested for HIV was 4.4\% $(10,830 / 247,006)$.

In order to determine factors that may be associated with HIV testing in an HCV infected population, a logistic regression was used to determine who was more likely to be tested.
Predictors of being tested: Table 2 presents the risk factors and general medical conditions that were used in these analyses. Many of the predictors were significant because of the overwhelming power of the large sample size. Only odds ratios above 1.20 (for the binary variables) were considered to be clinically significant.

The covariate of number of contacts with the hepatology clinic for HCV was also significant. The odds ratio of 1.025 indicated that each visit to the hepatology clinic increased the probability of the patient being tested for HIV by $2.5 \%$. The strongest predictor of being tested for HIV was being African-American (adj. OR $=1.74)$, those who had a visit for treatment of any substance use disorder $(\operatorname{adj} . \mathrm{OR}=1.65)$, those who had a visit for marijuana abuse/dependence (adj.

Table 2. Predictors of Being Tested for HIV in Logistic Regression

\begin{tabular}{|c|c|c|c|c|}
\hline & $\mathbf{B}^{*}$ & Standard Error & p Value & Odds Ratio \\
\hline \# of Hep C Visits & .026 & .000 & .000 & 1.027 \\
\hline Male ** & .000 & .030 & .991 & 1.000 \\
\hline African American ** & .552 & .012 & .000 & 1.736 \\
\hline Schizophrenia Visit ** & .148 & .016 & .000 & 1.160 \\
\hline Major Depression Visit ** & .152 & .012 & .000 & 1.164 \\
\hline PTSD Visit $* *$ & .079 & .012 & .000 & 1.082 \\
\hline Alcohol Use Disorder Visit ** & .105 & .018 & .000 & 1.111 \\
\hline Marijuana abuse/dependence Visit $* *$ & .242 & .013 & .000 & 1.273 \\
\hline Diabetes Visit $* *$ & .081 & .013 & .000 & 1.084 \\
\hline Hypertension Visit $* *$ & -.097 & .011 & .000 & .907 \\
\hline
\end{tabular}

${ }^{*} \mathrm{~B}=$ unstandardized regression weight

$* *=$ Dichotomous variable. 
$\mathrm{OR}=1.27)$ and those who had a visit for bipolar disorder (adj. OR =1.25). However alcohol use disorders alone (adj. $\mathrm{OR}=1.11$ ) was not as influential as the presence of any SUD.

Influence of HCV antiviral therapy on HIV testing: The influence of interferon- $\alpha$ antiviral therapy on HIV testing was also examined. Among the patients previously undiagnosed with HIV, 32,480 (13.6\%) were treated with interferon-alpha based antiviral therapy for hepatitis C. Over one third of veterans $(38.1 \%)$ who received antiviral therapy $(12,365 / 32,480)$ were tested for HIV prior to receiving interferon treatment, $13.4 \%(4,339 / 32,480)$ were tested after treatment, and $48.5 \%(15,776 / 32,480)$ were never tested during the observation period. The HIV positive rate among those tested before and after treatment was 1.8\% (334 veterans tested positive).

\section{DISCUSSION}

The Management of Hepatitis C National Institutes of Health Consensus Conference, 2002, as well as the VHA Treatment Guidelines for Patients with Chronic Hepatitis C, 2003, recommended that all people with HCV should be offered HIV testing and that people with HIV should be routinely screened for $\mathrm{HCV}$ infection [1, 18]. Moreover these guidelines also recommend that all people with $\mathrm{HCV}$ who are eligible for antiviral therapy be screened and tested for HIV prior to initiation of treatment. More recently the Centers for Disease Control (CDC) have modified their recommendations for HIV screening and testing in health care settings in order to enhance and improve case finding and treatment [19]. In 2003, the CDC introduced guidelines to make testing a routine part of medical care, on par with other diagnostic and screening tests. The CDC revised its guidelines in 2006 and recommended HIV screening for patients in all health-care settings after the patient is notified that testing will be performed unless the patient declines (opt-out screening) [20]. Additionally, they indicated that patients at high risk for HIV infection should be screened for HIV at least annually. Regarding consent, they recommended for the first time that separate written consent for HIV testing should not be required and that general consent for medical care should be considered sufficient to encompass consent for HIV testing. This also eliminated the requirement for prevention counseling with HIV diagnostic testing or screening [19]. The elimination of written informed consent and pre and post-test counseling for HIV in federal health care settings was implemented by the Department of Veterans Affairs in 2009. This change may reduce barriers to HIV testing and may serve to increase rates of testing among HCV positive veterans.

Our results suggest that HIV co-testing among patients with HCV was low. Slightly less than two thirds $(65 \%)$ of people in the HCV Clinical Case Registry were not tested for HIV. Additionally, $61.9 \%$ of those who received interferon therapy were not tested for HIV prior to beginning antiviral therapy. Given that $3.9 \%$ of the HCV CCR participants (who had been previously undiagnosed with HIV) tested positive during this observation period, it is likely that significant numbers of HIV cases among HCV positive veterans remain undetected. Our results are consistent with Huckans et al. (2005) which showed similar rates of testing among veterans in the northwest United States VHA facilities and clinics (VISN 20). The sample in this study was nationwide indicating that the low HIV co-testing rates is not isolated to one area of the country [17].

African-American veterans with $\mathrm{HCV}$ were more likely to be co- tested for HIV than other veterans. Veterans with severe mental illness and substance abuse were also more likely to be co-tested particularly if they had a SUD. This suggests that medical professionals may identify SUD as a proxy for risky sexual and injection practices. However, racial indicators may also be a particularly important factor in influencing this decision. This strategy for identifying atrisk individuals may miss many cases in which risk factors are not present or not known to the medical provider. Widespread opt-out testing for HIV is perhaps a better way to ensures early detection and treatment.

Our results also suggest that most veterans are not tested for HIV prior to antiviral treatment despite recommendations in treatment guidelines. Given the complexities of HIV coinfection and antiretroviral therapy [21] and the fact that HIV can suppress the immune system [22] knowledge of HIV status is paramount for optimal treatment outcomes.

The low rate of HIV testing has implications beyond those veterans with a positive $\mathrm{HCV}$ antibody test that has been confirmed by PCR to determine if the patient has active viremia. Gandhi et al. found that half of patients with new HIV diagnoses at VA hospitals had CD4 cell counts of less than 200 cells $/ \mathrm{mm}^{3}$ Also, half of the individuals with late presentation of HIV had received an average (median) of 6 physician visits at VA prior to being diagnosed with HIV, an indicator of missed opportunities for case finding. Since half of these veterans already had AIDS this suggests that early widespread screening is paramount to optimal healthcare [24].

Limitations: A strength of this study is the large and national sample of veterans who were enrolled in the VHA United States national HCV Clinical Case Registry. However, a major limitation of this study is the retrospective design with a dataset that was created from existing medical records. This approach presents potential problems since certain variables examined are incomplete and subject to clinician variability in case finding and documentation as well as presentation and history of the veteran. An example is race as a variable, which was unreported for $35 \%$ of the sample. Another limitation is that the sample contains only veterans who have been entered into the VHA HCV Clinical Case Registry and therefore does not include veterans who have not had their HCV antibody test confirmed by PCR testing or were not entered into the registry. Yet another limitation is that specific risk factors used in the in the logistic regression were based on chart diagnoses and did not necessarily meet specific diagnostic criteria. We did not have access to certain socioeconomic variables such as employment status, type of insurance, social support and access to methadone treatment programs. However most veterans in this clinical population do not work full time and many receive a pension for disability. All veterans in the HCV CCR are eligible for healthcare provided by VHA but some do have additional health insurance plans. Social support is variable. Most large VHA hospitals have 
methadone and substance abuse treatment programs as a part of standard services.

Finally, there may likely be variations among providers in the detection and documentation of risk factors such as IV drug use, treatment for sexually transmitted diseases, history of prostitution, and admission into methadone treatment programs. These factors may be much more salient to providers who order HIV tests for their patients [3]. Additional studies have considered some of these issues [23].

\section{CONCLUSION}

Our study suggests that significant barriers may exist within VHA facilities and clinics to HIV testing. Identifying and reducing these barriers will ultimately improve care for veterans with $\mathrm{HCV}$. Educating providers as well as veterans with HCV about the importance of being tested for HIV, particularly before starting antiviral therapy for $\mathrm{HCV}$, is also important and potentially life-saving as antiviral therapy can significantly reduce blood indices including white blood cell count. Integration of services across the VHA healthcare network is important in the care of veterans. Current research indicates that psychiatric and substance use disorders are common in veterans with HIV and HCV. Therefore integrating testing for HIV and HCV, health education and a team approach to treatment in primary care clinics, can eliminate barriers, and increase engagement for access to clinical assessment and treatment of HIV and HCV [24].

Integrated health care models are not only important in the management of psychiatric, cognitive and SUD issues faced by co-infected patients, but also help to address the additional social needs such as lack of housing, transportation and income. These social needs are more likely to present significant barriers to treatment and care management. Treatment in separate clinic locations or care that is not integrated facilitates poor compliance, treatment dropouts and poorer outcomes [25]. Careful monitoring of the clinical approach and quality control procedures will maximize chances that an integrated model develops and thus provides optimal services for this medically and psychologically complex population.

\section{ACKNOWLEDGEMENTS}

The authors wish to thank the national VA Hepatitis C Resource Center for general support of this project. We also wish to thank the many collaborators who have worked with us as well as the many veterans who have participated in hepatitis $\mathrm{C}$ related projects and research studies that we have conducted. In particular, we would like to thank Erick Dieperink, Jennifer Loftis, Matthew McQuesten, Benjamin Morasco and Brad Witke. Dr Hauser would like to thank Cathy, Anika, Jirina, Katia and Max Hauser, and Alba Pillwein for their continued support.

\section{ABBREVIATIONS}

$$
\begin{array}{ll}
\mathrm{B} & =\text { Unstandardized Regression Weight } \\
\mathrm{CCR} & =\text { Clinical Case Registry } \\
\mathrm{CDC} & =\text { Centers for Disease Control } \\
\mathrm{HCV} & =\text { Hepatitis C Virus }
\end{array}
$$

HIV = Human Immunodeficiency Virus

NAFLD $=$ Non Alcoholic Fatty Liver Disease

OR = Odds Ratio

PCR = Polymerase Chain Reaction

PTSD $=$ Posttraumatic Stress Disorder

$\mathrm{SE} \quad=$ Standard Error

SUD $=$ Substance Use Disorder

VISN $=$ Veteran Integrated Service Networks

VHA $=$ Veterans Health Administration

\section{REFERENCES}

[1] National Institutes of Health Consensus Development Conference Statement Management of hepatitis C 2002.

[2] Bräu N, Bini E, Shahidi A, et al. Prevalence of hepatitis c and coinfection with HIV among United States veterans in the New York metropolitan area. Am J Gastroenterol 2002; 97: 2071-8.

[3] Dominitz J, Boyko E, Koepsell T, et al. Elevated prevalence of hepatitis $\mathrm{C}$ infection in users of United States veterans medical centers. Hepatology 2005; 41: 88-96.

[4] Philips B, Mole L, Backus L, et al. Caring for veterans with HIV disease. Washington DC, Department of Veterans Affairs 2003.

[5] Valdiserri R, Rodriguez F, Holodniy, M. Frequency of HIV screening in the Veterans Health Administration: implications for early diagnosis of HIV infection. AIDS Educ Prev 2008; 20: 25864.

[6] Backus L, Boothroyd B, Deyton LR. HIV, hepatitis C and HIV/hepatitis C co-infection in vulnerable populations. AIDS 2005; 19: 513-9.

[7] Bini E, Currie S, Shen H, et al. National multicenter study of HIV testing and HIV seropositivity in patients with chronic hepatitis C virus infection. J Clin Gasteroenterol 2006; 40: 732-9.

[8] Andersson K, Chung R. Hepatitis C virus in the HIV-infected patient. Clin Liver Dis 2006; 10: 303-20.

[9] Rosenburg S, Goodman L, Osher F. Prevalence of HIV, hepatitis $\mathrm{B}$, and hepatitis $\mathrm{C}$ in people with severe mental illness. Am J Public Health 2001; 91: 31-7.

[10] Rosenberg S, Drake R, Brunette, M, et al. Hepatitis C virus and HIV co-infection in people with severe mental illness and substance use disorders. AIDS 2005; 19: S26-S33.

[11] Rockstroch JK, Spengler U. HIV and hepatitis c virus co-infection. Lancet 2004; 4: 437-44.

[12] Graham CS, Banden LR, Yu E, et al. Influence of human immunodeficiency virus infection on the course of hepatitis $\mathrm{C}$ virus infection: A meta-analysis. Clin Infect Dis 2001; 33: 562-9.

[13] Girardi E, Sabin CA, Monforte AD. Late diagnosis of HIV infection: Epidemiological features, consequences and strategies to encourage earlier testing. JAIDS 2007; 46: S3-S8.

[14] Rothman RE, Ketlogetswe KS, Dolan T, Wyer PC, Kelen GD. Preventive Care in the Emergency Department: Should Emergency Departments conduct routine HIV screening? A systematic review. Acad Emer Med 2003; 10: 278-85.

[15] Branson B Current HIV Epidemiology and Revised Recommendations for HIV Testing in Health-Care Settings. J Med Vir 2007; 79: S6-S10.

[16] Huckans MS, Blackwell AD, Harms TA, Indest DW, Hauser P. Integrated hepatitis $\mathrm{C}$ virus treatment: Addressing cormorbid substance use disorders and HIV infection. AIDS 2005; 19: S106S1 15.

[17] Wright TL, Yee H. VA treatment recommendations (version 5.0). In: Federal Practitioner, Edited by Veterans Health Administration 2003; 20(S5): 1-33.

[18] Centers for Disease Control. Revised guidelines for HIV counseling, testing, and referral. MMWR 2001; 50 (No. RR-19): 162.

[19] Centers for Disease Control (2006). Revised recommendations for HIV testing of adults, adolescents, and pregnant women in healthcare settings. Recommendations and Reports. September 22, 2006/55 (RR14); 1-17. http://www.cdc.gov/mmwr/preview/mmwr $\mathrm{html} / \mathrm{rr} 5514 \mathrm{a} 1 . \mathrm{htm}$ 
[20] Borghi V, Puoti M, Mussini C, et al. HIV coinfection and antiretroviral therapy enhances liver steatosis in patients with hepatitis $\mathrm{C}$, but only in those infected by HCV genotype other than 3. Antivir Ther. 2008; 13(8): 1057-65.

[21] Loftis JM, Hauser P. Treating Hepatitis C in Patients with Comorbid Psychiatric and Substance Use Disorders. Dir Psych 2008; 28(4): 227-243

[22] Goetz MB, Bowman C, Hoang $\mathrm{T}$, et al. Implementing and evaluating a regional strategy to improve testing rates in VA patients at risk for HIV, utilizing the QUERI process as a guiding framework: QUERI Series. Implement Sci 2008: 3-16
[23] Gandhi NR, Skanderson M, Gordon KS. Concato J, Justice AC. Delayed presentation for human immunodeficiency virus (HIV) care among Veterans. Med Care 2007; 45: 1105-9.

[24] Rifai MA, Moles JK, Lehman LP, Van Der Linde BJ. Hepatitis C screening and treatment outcomes in patients with substance use/dependent disorders. Psychosom 2006; 47: 112-21.

[25] Willenbring ML. Integrating care for patients with infectious, psychiatric, and substance abuse disorders: Concepts and approaches. AIDS 2005; S3; s227-s237.

(C) Fuller et al.; Licensee Bentham Open.

This is an open access article licensed under the terms of the Creative Commons Attribution Non-Commercial License (http://creativecommons.org/licenses/ by-nc/3.0/) which permits unrestricted, non-commercial use, distribution and reproduction in any medium, provided the work is properly cited. 